\title{
Can IASLC/ATS/ERS subtype help predict response to chemotherapy in small biopsies of advanced lung adenocarcinoma?
}

\author{
Gregory J. Riely ${ }^{1}$ and William D. Travis ${ }^{2}$
}

Affiliations: 'Thoracic Oncology Service, Dept of Medicine, Memorial Sloan Kettering Cancer Center, New York, NY, and ${ }^{2}$ Dept of Pathology, Memorial Sloan Kettering Cancer Center, New York, NY, USA.

Correspondence: G.J. Riely, Thoracic Oncology Service, Memorial Sloan Kettering Cancer Center, 300 East 66th Street, New York, NY 10065, USA. E-mail: rielyglamskcc.org

0

@ERSpublications

The possibility of using tumour histology as a predictive marker is exciting: further validation is required http://ow.ly/uFkdM

The identification of somatic EGFR mutations and $A L K$ gene rearrangements has dramatically changed our understanding of the biology of lung cancers and provided extraordinarily helpful predictive biomarkers that allow physicians to determine which patients will have the greatest likelihood of benefit with certain targeted therapies. These predictive biomarkers allow rational use of the targeted therapies appropriate for the individual driver oncogene (erlotinib, gefitinib, and afatinib for EGFR mutant cancers, and crizotinib for $A L K$ rearranged cancers). There are even some indications that these mutations predict for better outcomes with some standard chemotherapy $[1,2]$. Despite this, the majority of patients either have no identifiable driver oncogene or it is one without an associated effective therapy (e.g. KRAS mutation). For these patients, it is challenging to decide which therapy should be given. The difficulty arises from the poor response rates for conventional chemotherapy regimens, with just $30-35 \%$ of patients having significant tumour shrinkage with state-of-the art first line platinum-based chemotherapy regimens. Clearly, there is a desperate need for further insight into therapy choices and biology for the majority of patients with lung adenocarcinomas.

In this issue of European Respiratory Journal, CAMPOS-PARRA et al. [3] present findings that suggest that using the approach to predominant pathological subtyping proposed in the International Association for the Study of Lung Cancer (IASLC)/American Thoracic Society (ATS)/European Respiratory Society (ERS) classification of lung adenocarcinoma in patients with advanced disease may predict outcomes after standard chemotherapy. The IASLC/ATS/ERS classification, published in 2011, provides an overall systematic approach to analysis of lung adenocarcinomas [4]. This classification provides recommendations for molecular analysis, a classification system for resection specimens, and guidance for evaluation of small biopsy specimens. Resected invasive adenocarcinomas are classified based on the predominant pattern, leading to the major subtypes of lepidic predominant (formerly nonmucinous bronchioloalveolar carcinoma), acinar predominant, papillary predominant, micropapillary predominant or solid predominant. Since the introduction of this classification, its use in resection specimens as well as its association with recurrence and survival after resection for early stage disease has been reported by several groups [5-8]. In these series, lower grade tumours (such as lepidic predominant) have been shown to be associated with a lower risk for recurrence, while those with higher grade tumours (such as micropapillary, invasive mucinous adenocarcinoma and solid predominant tumours) had worse disease-free and overall survival [9]. This prognostic ability remains significant in multivariate analyses that include factors such as age, stage and

Received: March 132014 | Accepted: March 132014

Conflict of interest: None declared.

Copyright @ERS 2014 
resection margin status, among other covariates, acting as an independent predictor of disease-free and overall survival [6, 10-12]. However, in some series, its prognostic ability has been weaker [13].

In this context, CAMPOS-PARRA et al. [3] report their evaluation of small tumour biopsies from patients with advanced lung adenocarcinomas using the new IASLC/ATS/ERS lung adenocarcinoma classification system. In a group of patients with advanced lung adenocarcinoma treated with standard first-line chemotherapy regimens, they found that high grade tumours (in this series: micropapillary, papillary and solid predominant), as compared with lower grade tumours, have a higher response rate (37\% versus $25 \%$, $\mathrm{p}=0.034)$, progression-free survival $(6.4$ versus 5.5 months, $\mathrm{p}=0.009)$ and overall survival $(25$ versus 17 months, $p=0.023)$. The better outcomes for those with high grade tumours stand in stark contrast to the outcomes observed in numerous series of patients with resected lung adenocarcinomas where high grade adenocarcinoma histology is associated with poorer survival. The intriguing possibility raised by these data is that the standard platinum-based doublet chemotherapy used to treat nonsmall cell lung cancer is more effective in higher grade tumours. Potentially validating data was recently presented as an abstract from the LACE-BIO study in which resected lung adenocarcinomas classified as solid and micropapillary subtypes showed a significantly higher benefit from adjuvant chemotherapy [14]. There are certainly parallels for this concept in several tumour types. Notably, low grade lymphomas, such as follicular lymphoma, are chemotherapy-responsive but are not considered curable with current treatments; by contrast, more aggressive lymphomas can be cured with combination chemotherapy.

A critically important caveat to our understanding of these data is that this study by CAMPOS-PARRA et al. [3] classifies tumours from patients with metastatic disease solely based upon a single biopsy result with a small tumour sampling. Often, this is the only material available for determination of diagnosis and decisions regarding treatment. Traditionally, the diagnosis of advanced lung cancer has been based on small biopsy and cytology specimens. These specimens are a small portion of the tumour and subject to a sampling bias, which could lead to an incomplete classification (e.g. biopsy of an adenosquamous carcinoma could reveal only the adenocarcinoma component or the squamous component). Often, there is inadequate material to clearly classify the tumour by light microscopy alone and immunohistochemistry may be required to provide a more clear diagnosis. In the 2011 IASLC/ATS/ERS lung adenocarcinoma classification system, there is a nomenclature for small biopsy specimens in which histology alone is inadequate to describe the tumour. Such tumours, after use of immunohistochemistry, can sometimes be described as nonsmall cell lung cancer (NSCLC), favour adenocarcinoma (if an adenocarcinoma specific marker, such as TTF-1 or Napsin A, is positive) or NSCLC, favour squamous (if a squamous marker such as p40 is positive). This approach helps to sort out whether a tumour is an adenocarcinoma or squamous cell carcinoma. Even with small samples, one would expect the predominant subtype to be present in small biopsies in the majority of patients. However, it is likely that small biopsy samples will not always result in an accurate classification of the predominant subtype. This issue may provide further impetus to obtain small samples with substantial amounts of tissue, as can be obtained with core biopsies.

There are clear limitations to the data presented, which prevent the immediate use of this information in clinical practice. The patients in this series were not typical of patients with advanced lung adenocarcinoma: there were more women, more people without a history of cigarette smoking and other, smaller differences. As part of their chemotherapy regimens, these patients were not treated with either pemetrexed or bevacizumab, two commonly used agents. Patients with EGFR mutations were generally detected after they had completed all treatment; therefore, patients were not routinely treated with EGFR tyrosine kinase inhibitors which could have marked effects on overall survival, potentially changing outcomes for some subgroups. While examination of surgically resected tumours allows a full evaluation of tumour architecture and complete classification of the tumour, in advanced lung cancer a small biopsy specimen is typically the sole source for diagnosis, classification and molecular analysis.

Despite the limitations raised here and in the paper, the possibility of using tumour histology as a potential predictive marker is exciting. It will be important to validate these findings with a group of patients who are more similar to average lung cancer patients and, in addition, those who have received more modern chemotherapy regimens. Validation of the IASLC/ATS/ERS classification in small biopsies from patients with advanced lung cancer as a step toward stratification of patients regarding platinum-based therapies could lead to dramatic changes in how and when chemotherapy is administered. If this can be validated in other retrospective cohorts, it would be worth testing in a prospective clinical trial. 


\section{References}

1 Shaw AT, Kim DW, Nakagawa K, et al. Crizotinib versus chemotherapy in advanced ALK-positive lung cancer. N Engl J Med 2013; 368: 2385-2394.

2 Mok TS, Wu YL, Thongprasert S, et al. Gefitinib or carboplatin-paclitaxel in pulmonary adenocarcinoma. $N$ Engl J Med 2009; 361: 947-957.

3 Campos-Parra AD, Avilés A, Contreras-Reyes S, et al. Relevance of the novel IASLC/ATS/ERS classification of lung adenocarcinoma in advanced disease. Eur Respir J 2014; 43: 1439-1447.

4 Travis WD, Brambilla E, Noguchi M, et al. International Association for the Study of Lung Cancer/American Thoracic Society/European Respiratory Society: international multidisciplinary classification of lung adenocarcinoma. J Thorac Oncol 2011; 6: 244-285.

5 Tsuta K, Kawago M, Inoue E, et al. The utility of the proposed IASLC/ATS/ERS lung adenocarcinoma subtypes for disease prognosis and correlation of driver gene alterations. Lung Cancer 2013; 81: 371-376.

6 Russell PA, Barnett SA, Walkiewicz M, et al. Correlation of mutation status and survival with predominant histologic subtype according to the new IASLC/ATS/ERS lung adenocarcinoma classification in stage III (N2) patients. J Thorac Oncol 2013; 8: 461-468.

7 Yoshizawa A, Sumiyoshi S, Sonobe M, et al. Validation of the IASLC/ATS/ERS lung adenocarcinoma classification for prognosis and association with EGFR and KRAS gene mutations: analysis of 440 Japanese patients. $J$ Thorac Oncol 2013; 8: 52-61.

8 Yoshizawa A, Motoi N, Riely GJ, et al. Impact of proposed IASLC/ATS/ERS classification of lung adenocarcinoma: prognostic subgroups and implications for further revision of staging based on analysis of 514 stage I cases. Mod Pathol 2011; 24: 653-664.

9 Kadota K, Colovos C, Suzuki K, et al. FDG-PET SUVmax combined with IASLC/ATS/ERS histologic classification improves the prognostic stratification of patients with stage I lung adenocarcinoma. Ann Surg Oncol 2012; 19: 3598-3605.

10 Song Z, Zhu H, Guo Z, et al. Prognostic value of the IASLC/ATS/ERS classification in stage I lung adenocarcinoma patients-based on a hospital study in China. Eur J Surg Oncol 2013; 39: 1262-1268.

11 Yanagawa N, Shiono S, Abiko M, et al. New IASLC/ATS/ERS classification and invasive tumor size are predictive of disease recurrence in stage I lung adenocarcinoma. J Thorac Oncol 2013; 8: 612-618.

12 Hung JJ, Jeng WJ, Chou TY, et al. Prognostic value of the new International Association for the Study of Lung Cancer/American Thoracic Society/European Respiratory Society lung adenocarcinoma classification on death and recurrence in completely resected stage I lung adenocarcinoma. Ann Surg 2013; 258: 1079-1086.

13 Westaway DD, Toon CW, Farzin M, et al. The International Association for the Study of Lung Cancer/American Thoracic Society/European Respiratory Society grading system has limited prognostic significance in advanced resected pulmonary adenocarcinoma. Pathology 2013; 45: 553-558.

14 Brambilla EM, Marguet S, Le Teuff G, et al. Prognostic and predictive value of a new IASLC/ATS/ERS lung adenocarcinoma classification in a pooled analysis of four adjuvant chemotherapy trials: a LACE-BIO study. J Thorac Oncol 2013; 8: Suppl. 2, S201. 